International Journal of Bifurcation and Chaos, Vol. 10, No. 8 (2000) 1787-1804

(c) World Scientific Publishing Company

\title{
BIFURCATION DYNAMICS OF THREE-DIMENSIONAL SYSTEMS
}

\author{
PAUL E. PHILLIPSON* and PETER SCHUSTER \\ Institut für Theoretische Chemie und Molekulare Strukturbiologie der Universität Wien, \\ Währingerstraße 17, A-1090 Vienna, Austria
}

Received June 16, 1999; Revised October 22, 1999

\begin{abstract}
Oscillations described by autonomous three-dimensional differential equations display multiple periodicities and chaos at critical parameter values. Regardless of the subsequent scenario the key instability is often an initial bifurcation from a single period oscillation to either its subharmonic of period two, or a symmetry breaking bifurcation. A generalized third-order nonlinear differential equation is developed which embraces the dynamics vicinal to these bifurcation events. Subsequently, an asymptotic averaging formalism is applied which permits an analytic development of the bifurcation dynamics, and, within quantifiable limits, prediction of the instability of the period one orbit in terms of the system control parameters. Illustrative applications of the general formalism, are made to the Rössler equations, Lorenz equations, three-dimensional replicator equations and Chua's circuit equations. The results provide the basis for discussion of the class of systems which fall within the framework of the formalism.
\end{abstract}

\section{Introduction}

Autonomous three-dimensional differential equation systems of the form

$$
\frac{d \mathbf{X}}{\mathbf{d t}}=\mathbf{F}(\mathbf{X}) \text { with } \mathbf{F}(\mathbf{X})=\left(F_{1}(\mathbf{X}), F_{2}(\mathbf{X}), F_{3}(\mathbf{X})\right.
$$

and

$$
\mathbf{X}=\left(X_{1}, X_{2}, X_{3}\right)
$$

can display a rich diversity of periodic, multiple periodic and chaotic flows dependent upon the specific values of one or more control parameters. The control parameter, or a combination of parameters suitable to describe the bifurcation of interest, is denoted by $r$. Analysis of the equations linearized around a fixed point $\mathbf{x}_{\mathbf{o}}$ such that $\mathbf{F}\left(\mathbf{x}_{\mathbf{o}}\right)=0$ serve to provide local solutions which evolve as $e^{\sigma t}$. These solutions are stable or unstable depending upon whether or not roots $\sigma=\sigma_{k}(r), k=1,2,3$, of the secular equation for the eigenvalues of the Jacobian

$$
\begin{aligned}
\left(\mathbf{J}=\left\{J_{i j}=\partial F_{i}(\mathbf{X}) / \partial X_{j}\right\}\right), \\
\sigma^{3}+A_{2}(r) \sigma^{2}+A_{1}(r) \sigma+A_{0}(r)=0,
\end{aligned}
$$

are all either negative or have negative real parts. The transition from stability to instability occurs when the real part of one or more of the eigenvalues passes through a zero from negative to positive. The prediction of critical values of the control parameters at which such bifurcations occur is, in principle, complete and unambiguous as long as the functional dependencies $A_{k}(r)$ are known. This analysis, limited as it is to a local description around the fixed point, provides no global information about how, or the subsequent control parameter conditions when, unstable solutions evolve into the myriad of possible oscillatory behaviors. In many cases the nonlinear terms, ignored in the construction of an unstable linearized solution, drive the system into a limit cycle, or period one orbit. However, the unique feature of three-dimensional

*Address for correspondence: Department of Physics, Box 390, University of Colorado Boulder, CO 80309, USA.

E-mail: phillipe@spot.colorado.edu 
systems is that period one orbits themselves become unstable, and once this happens multiple periodicity and chaos are usually inevitable with a scenario specific to the system. Such bifurcation structures can often be explored with a combination of analytical and computer-assisted numerical techniques [Kubiček \& Marek, 1983]. The scenario is quite generally initiated by an instability of the period one orbit which bifurcates into an orbit of period two ( $1 \rightarrow 2$ bifurcation). This designation will be used here to specify either a period doubling bifurcation or a symmetry breaking bifurcation where a limit cycle becomes unstable to produce two stable limit cycles. The purpose of the present work is to provide an analytic formalism for quantitative prediction as to how, and the control parameter conditions when, $1 \rightarrow 2$ bifurcations can occur. By so doing one can create a bridge between a quasi-static bifurcation from a fixed point, quantified by fixed point analysis, and a dynamical bifurcation of orbits as heralded by this bifurcation event. It will also be quantified by an equivalent to fixed point analysis. In the present case the fixed point is approximated as the constant amplitude of a period two orbit which is self-consistently created by its precursor period one orbit. The period two bifurcation occurs at the birth of this amplitude, at which point the period one orbit itself becomes unstable. Fixed point analysis local to this amplitude provides the conditions under which the period two orbit persists until it becomes unstable as well. The development generalizes a previous study [Phillipson \& Schuster, 1998] of the bifurcation structure of a prototypic dynamical system [Arneodo et al., 1985]. The next three sections will provide the development of the mathematical formalism as well as an analysis of its limitations. Section 5 will show illustrative applications to four canonical dynamical systems: the Rössler equations, Lorenz equations, three-dimensional replicator equations and Chua's circuit equations. The final section provides, in light of these limitations, an assessment of an acceptable class of dynamical systems as illustrated by these examples.

\section{The Bifurcation Equations}

Physical and chemical systems of interest modeled by Eq. (1) are usually encapsulated by the functions $\mathbf{F}$ which are polynomial in the coordinates. Then, the differential equations can be partitioned according to

$$
\begin{aligned}
\mathbf{X} & =\mathbf{X}^{(\mathbf{1})}+\mathbf{X}^{(\mathbf{2})} \text { with } \mathbf{F}(\mathbf{X}) \\
& =\mathbf{F}\left(\mathbf{X}^{(\mathbf{1})}\right)+\mathbf{G}\left(\mathbf{X}^{(\mathbf{1})}, \mathbf{X}^{(\mathbf{2})}\right) \\
\frac{d \mathbf{X}^{(\mathbf{1})}}{d t} & =\mathbf{F}\left(\mathbf{X}^{(\mathbf{1})}\right)\left[\begin{array}{l}
\text { equation for the } \\
\text { period one orbit }]
\end{array}\right.
\end{aligned}
$$

$$
\begin{array}{r}
\frac{d \mathbf{X}^{(2)}}{d t}=\mathbf{G}\left(\mathbf{X}^{(\mathbf{1})}(\omega t), \mathbf{X}^{(\mathbf{2})}\right) \text { [equation for } \\
\text { the period two orbit] }
\end{array}
$$

It is assumed that $\mathbf{X}^{(\mathbf{1})}(\omega t)$ is the period one solution of period $T=2 \pi / \omega$ to Eq. (1) in a parameter region in which it is stable. In this region $\mathbf{X}^{(\mathbf{2})}=0$, necessarily. The orbit $\mathbf{X}^{(\mathbf{1})}$ loses stability at those bifurcation points in parameter space where $\mathbf{X}^{(\mathbf{2})}$ is born. Vicinal to a $1 \rightarrow 2$ bifurcation point the latter contributes a period two correction $\mathbf{X}^{(2)}(\omega t / 2)$. Equations (1), cast into the form of Eq. (2), express the assumption of the present work that the period one solution, $\mathbf{X}^{(\mathbf{1})}(t)$, functions as the driving force for the creation of the period two solution, formally similar to two-dimensional systems driven by an external driving force [Jackson, 1991]. The complications of doubling the number of equations and introducing the requirement of explicit time dependence for the determination of the period two orbit is compensated by facilitating an hierarchical procedure for analysis of bifurcations which builds upon the application of asymptotic averaging techniques originally introduced by Krylov and Bogoliubov [1947]. Similarities to the present approach is the application of this averaging procedure to the study of bifurcation structures of the two-dimensional Duffing's equation driven by an external force of a single frequency [Holmes \& Holmes, 1981]. Here, however, the driving force is a period one orbit generated internally by the dynamics of the autonomous system Eq. (2b), which destroys its own stability in the process of generating the period two orbit.

Application of asymptotic methods requires transformation of Eq. (1) into a normal form such that it is possible to effect the averaging procedure. We assume formally the existence of a transformation from the system coordinates $\left[X_{1}, X_{2}, X_{3}\right]$ to dynamical coordinates $[x, y, z]$ such that the three first-order differential equations (1) can be expressed as a single third-order differential equation 
according to

$$
X_{k}=X_{k}(x, y, z), \quad k=1,2,3,
$$

with

$$
\frac{d x}{d t}=y, \frac{d y}{d t}=z, \text { and } \frac{d z}{d t}=W(x, y, z)
$$

If Eq. (1) were to be linearized around a fixed point, choosing $X_{k}=x$ for any $k$, one obtains $W=g_{1} x+g_{2} y+g_{3} z$, with $\left[g_{1}=\sigma_{1} \sigma_{2} \sigma_{3}, g_{2}=\right.$ $-\left(\sigma_{1} \sigma_{2}+\sigma_{2} \sigma_{3}+\sigma_{1} \sigma_{3}\right)$, and $\left.g_{3}=\sigma_{1}+\sigma_{2}+\sigma_{3}\right]$, where $\sigma_{k}, k=1,2,3$, are the eigenvalues of the secular equation. For the complete nonlinear system a generic global transformation does not exist. In practice, however, such a transformation can be found specific to the system. The resultant expression for $W$ is often very complex and sensitive to a choice of transformation which itself may not be unique. On the other hand, the information sought is restricted to the environment of a period one orbit, which suggests approximation of $W$ as the sum of a linear and a quadratic form according to

$$
\begin{aligned}
\frac{d z}{d t}= & g_{1} x+g_{2} y+g_{3} z+h+Q(x, y, z) \\
Q(x, y, z)= & q_{11} x^{2}+q_{12} x y+q_{13} x z \\
& +q_{22} y^{2}+q_{23} y z+q_{33} z^{2} \\
y= & \frac{d x}{d t}, \quad z=\frac{d y}{d t}
\end{aligned}
$$

where $g_{k}, h$ and $q_{i j}$ are constants which are to be related to the control parameters of Eq. (1). Equations $(3 \mathrm{a})$ and $(3 \mathrm{~b})$ represent transformation to a normal form and Eq. (4) is formally a power series expansion in the coordinates through quadratic terms. This approximation usually, but not always, destroys globally the attractor, but locally can provide a generic picture of $1 \rightarrow 2$ dynamics as the next step after the formation of the period one orbit arising from instability of an attracting fixed point.

Questions of stability and bifurcations of various kinds of normal forms have led to formal analytical results, without specific application, in terms of assumed variables defined on a Poincaré surface of section [Iooss \& Joseph, 1990]. While the present formulation does not yet have a similar rigorous theoretical foundation, nevertheless Eqs. (3a), (3b) and (4) will serve here as building blocks to formulate bifurcation dynamics in terms of an operational construction which on the one hand is applicable to a class of autonomous three-dimensional systems and on the other can reflect the particularities of a chosen system. In so doing quantitative predictions of bifurcation events can be made within the context of the dynamics specific to this choice. The examples in Sec. 5 provide the basis for Sec. 6 which will address the more general question of the determination of what class of systems can be addressed by the present scheme.

\section{The Averaging Procedure}

In accordance with the decomposition of Eqs. (2a)(2c) the solutions of Eq. (4) will be expressed as the sum of two terms $x=x_{1}+x_{2}, y=y_{1}+y_{2}$, $z=z_{1}+z_{2}$ where $x_{1}$ is considered to be a period one orbit which must evolve as Eq. (4),

$$
\begin{array}{r}
\frac{d z_{1}}{d t}=g_{1} x_{1}+g_{2} y_{1}+g_{3} z_{1}+h+Q\left(x_{1}, y_{1}, z_{1}\right) \\
\text { [equation for the period one orbit] }
\end{array}
$$

so that $x_{2}$ evolves according to

$$
\begin{aligned}
\frac{d z_{2}}{d t}= & F_{1}(t) x_{2}+F_{2}(t) y_{2}+F_{3}(t) z_{2}+Q\left(x_{2}, y_{2}, z_{2}\right) \\
F_{1}(t)= & g_{1}+2 q_{11} x_{1}(t)+q_{12} y_{1}(t)+q_{13} z_{1}(t) \\
F_{2}(t)= & g_{2}+2 q_{22} y_{1}(t)+q_{12} x_{1}(t)+q_{23} z_{1}(t) \quad(5 \mathrm{~b}) \\
F_{3}(t)= & g_{3}+2 g_{33} z_{1}(t)+q_{13} x_{1}(t)+q_{23} y_{1}(t) \\
& \text { [equation for the period two orbit] }
\end{aligned}
$$

To proceed analytically, the period one orbit will be approximated by a simple harmonic form with the neglect of higher harmonics. Substitution into Eq. (5a) results in the following expression for the period one limit cycle

$$
\begin{gathered}
x_{1}(t)=D_{0}+D_{1} \cos (\omega t) \\
q_{12} q_{13} D_{0}^{2}+\left(2 q_{11}+g_{2} q_{13}+g_{3} q_{12}\right) D_{0} \\
+\left(g_{1}+g_{2} g_{3}\right)=0 \\
D_{1}^{2}=\frac{-2\left(g 1 D_{0}+q_{11} D_{0}^{2}+h\right)}{q_{11}+\omega^{2}\left(q_{22}-q_{13}\right)} \\
\text { with } \quad \omega^{2}=-\left(g_{2}+q_{12} D_{0}\right)
\end{gathered}
$$

where, consistent with this harmonic approximation a contribution of the coefficient $q_{33}$ to $D_{1}$ is neglected. The amplitude factors $D_{0,1}$ and frequency $\omega$ in terms of the differential equation parameters according to Eq. (6b) are determined by setting the resultant constant term and coefficients of $\cos (\omega t)$, 
$\sin (\omega t)$ independently equal to zero. The neglect of higher harmonic terms in $2 \omega t$ is consistent with the single harmonic approximation of the period one orbit. Substitution of Eq. (6a) in Eq. (5b) introduces explicit time dependence through the functions $F$ into the nonautonomous equation for $x_{2}$ according to

$$
\begin{gathered}
F_{1}(t)=A_{0}+A_{1} \cos (\omega t)+A_{2} \sin (\omega t) \\
F_{2}(t)=B_{0}+B_{1} \cos (\omega t)+B_{2} \sin (\omega t) \\
F_{3}(t)=C_{0}, \quad C_{0}<0 \\
A_{0}=g_{1}+2 q_{11} D_{0}, \quad A_{1}=\left(2 q_{11}-\omega^{2} q_{13}\right) D_{1} \\
A_{2}=-\omega q_{12} D_{1} \quad B_{0}=g_{2}+q_{12} D_{0} \\
B_{1}=\left(q_{12}-\omega^{2} q_{23}\right) D_{1}, \quad B_{2}=-2 \omega q_{22} D_{1} \\
C_{0}=g_{3}+q_{13} D_{0}
\end{gathered}
$$

The term $z_{2}$ in Eq. (5b) provides the stabilizing factor of dissipation which must be negative. This condition imposes the neglect of oscillatory contributions to $F_{3}$. Parallel to two-dimensional studies [Holmes \& Holmes, 1981], the period doubled orbit $x_{2}(\omega t / 2)$ is constructed according to the following van der Pol transformation to functions $u, v$ defined by

$$
\begin{aligned}
x_{2}= & u \cos \left(\frac{\omega t}{2}\right)-v \sin \left(\frac{\omega t}{2}\right) \\
y_{2}= & -\frac{\omega}{2} u \sin \left(\frac{\omega t}{2}\right)-\frac{\omega}{2} v \cos \left(\frac{\omega t}{2}\right) \\
z_{2}= & \left(\left(\frac{\omega}{2}\right)^{2} u+\frac{\omega}{2} \frac{d v}{d t}\right) \cos \left(\frac{\omega t}{2}\right) \\
& +\left(\left(\frac{\omega}{2}\right)^{2} v-\frac{\omega}{2} \frac{d u}{d t}\right) \sin \left(\frac{\omega t}{2}\right)
\end{aligned}
$$

for which the inverse transformation is

$$
\begin{gathered}
u=x_{2} \cos \left(\frac{\omega t}{2}\right)-\frac{2 y_{2}}{\omega} \sin \left(\frac{\omega t}{2}\right) \\
v=-x_{2} \sin \left(\frac{\omega t}{2}\right)-\frac{2 y_{2}}{\omega} \cos \left(\frac{\omega t}{2}\right) \\
{\left[\begin{array}{l}
\frac{d u}{d t} \\
\frac{d v}{d t}
\end{array}\right]=-\left[\frac{\omega x_{2}}{2}+\frac{2 z_{2}}{\omega}\right]\left[\begin{array}{l}
\sin \left(\frac{\omega t}{2}\right) \\
\cos \left(\frac{\omega t}{2}\right)
\end{array}\right]}
\end{gathered}
$$

Equation (9c) is a consequence of Eqs. (8a) and (8b) consistent with the requirement that $y_{2}=d x_{2} / d t$.
Differentiation of the last equations gives

$$
\left[\begin{array}{l}
\frac{d^{2} u}{d t^{2}}-\frac{\omega}{2} \frac{d v}{d t} \\
\frac{d^{2} v}{d t^{2}}+\frac{\omega}{2} \frac{d u}{d t}
\end{array}\right]=-\left[\frac{\omega y_{2}}{2}+\frac{2}{\omega} \frac{d z_{2}}{d t}\right]\left[\begin{array}{c}
\sin \left(\frac{\omega t}{2}\right) \\
\cos \left(\frac{\omega t}{2}\right)
\end{array}\right]
$$

Substitution of Eq. (5b) in Eq. (10), then with the use of Eq. (7) one obtains

$$
\begin{aligned}
\frac{d^{2} u}{d t^{2}}= & A_{11}(t) \frac{d u}{d t}+A_{12}(t) \frac{d v}{d t}+A_{13}(t) u \\
& +A_{14}(t) v+U(u, v, \ldots, t) \\
\frac{d^{2} v}{d t^{2}}= & A_{21}(t) \frac{d u}{d t}+A_{22}(t) \frac{d v}{d t}+A_{23}(t) u \\
& +A_{24}(t) v+V(u, v, \ldots, t)
\end{aligned}
$$

with

$$
\begin{aligned}
A_{i j}(t) & =c_{i j}+g_{i j}(t) \text { and } g_{i j}(t) \\
& =a_{i j} \cos (\omega t)+b_{i j} \sin (\omega t)
\end{aligned}
$$

where $U$ and $V$ are functions composed of linear combination of all products of the functions $[u, v$, $d u / d t, d v / d t]$ each term of which is multiplied by time-periodic coefficients $[\cos (n \omega t / 2), \sin (n \omega t / 2)$, $n=1,3]$. The $\left[c_{i j}, a_{i j}, b_{i j}\right]$ constants of the oscillating coefficients $A_{i j}$ are given in Table 1. Suppose $\mathbf{x} \in R^{2}$ such that $d^{2} \mathbf{x} / d t^{2}=\mathbf{F}(d \mathbf{x} / d t, \mathbf{x}, t)$ where $\mathbf{F}$ is $T$-periodic in $t$. The solutions of Eq. (11) will be approximated by the solution of the averaged differential equations defined by

$$
\frac{\overline{d^{2} \mathbf{x}}}{d t^{2}} \equiv \frac{1}{T} \int_{0}^{T} \mathbf{F}\left(\frac{d \mathbf{x}}{d t}, \mathbf{x}, t\right) d t .
$$

Here $\mathbf{x}=(u, v)$ with $T=4 \pi / \omega$. Upon carrying out the averaging procedure for Eq. (11) the functions $U, V$ quadratic in the coordinate average to zero and the only coefficients that survive for the linear terms are the quantities $c_{i j}$. The results are linearly coupled equations in $u, v$ whose time dependencies are of the form $e^{\sigma t}$ where $\sigma$ are roots of a secular equation with necessarily real parts. The solutions either regress to zero or are unstable. In any case there are no legitimate finite solutions in this approximation. The construction of acceptable bifurcating period two solutions requires extension of the asymptotic averaging procedure which will 
Table 1. Coefficients $A_{i j}=\left[c_{i j}, a_{i j}, b_{i j}\right]$ of Eq. (11) in terms of System Parameters Eq. (7).

$$
\begin{aligned}
& c_{11}=\frac{C_{0}}{2} \quad a_{11}=-\frac{C_{0}}{2} \quad b_{11}=0 \\
& c_{12}=\frac{\omega}{2} \quad a_{12}=0 \quad b_{12}=\frac{C_{0}}{2} \\
& c_{21}=-\frac{\omega}{2} \quad a_{21}=0 \quad b_{21}=\frac{C_{0}}{2} \\
& c_{22}=\frac{C_{0}}{2} \quad a_{22}=\frac{C_{0}}{2} \quad b_{22}=0 \\
& c_{13}=\frac{\omega^{2}}{8}+\frac{B_{0}}{2}-\frac{A_{2}}{2 \omega}-\frac{B_{1}}{4} \quad a_{13}=\frac{1}{2}\left(B_{1}-B_{0}\right)-\frac{\omega^{2}}{8} \quad b_{13}=-\frac{A_{0}}{\omega}+\frac{B_{2}}{2}+\frac{\omega C_{0}}{4} \\
& c_{14}=\left[\frac{A_{0}}{\omega}+\frac{B_{2}}{4}\right]-\left[\frac{A_{1}}{2 \omega}+\frac{\omega C_{0}}{4}\right] \quad a_{14}=\frac{\left(A_{1}-A_{0}\right)}{\omega}+\frac{\omega C_{0}}{4} \quad b_{14}=\frac{A_{2}}{\omega}+\frac{B_{0}}{2}+\frac{\omega^{2}}{8} \\
& c_{23}=\left[\frac{\omega C_{0}}{4}+\frac{B_{2}}{4}\right]-\left[\frac{A_{0}}{\omega}+\frac{A_{1}}{2 \omega}\right] \quad a_{23}=\frac{\omega C_{0}}{4}-\frac{A_{0}+A_{1}}{\omega} \quad b_{23}=-\frac{A_{2}}{\omega}+\frac{B_{0}}{2}+\frac{\omega^{2}}{8} \\
& c_{24}=\frac{\omega^{2}}{8}+\frac{B_{0}}{2}+\frac{A_{2}}{2 \omega}+\frac{B_{1}}{4} \quad a_{24}=\frac{B_{0}+B_{1}}{2}+\frac{\omega^{2}}{8} \quad b_{24}=\frac{A_{0}}{\omega}+\frac{B_{2}}{2}-\frac{\omega C_{0}}{4}
\end{aligned}
$$

most importantly include the effects of nonvanishing quadratic terms in $U, V$. These functions provide the mechanism which stabilizes the period two orbit over a prescribed control parameter range.

Accordingly, the next stage is to introduce the following second transformation from $(u, v)$ to new coordinates $(y, z)$ defined by generating functions $w_{1}, w_{2}$ to be determined

$$
\begin{gathered}
u=y+w_{1}\left(y, z, \frac{d y}{d t}, \frac{d z}{d t}, t\right), \\
v=z+w_{2}\left(y, z, \frac{d y}{d t}, \frac{d z}{d t}, t\right) \\
\frac{d u}{d t}=\frac{d y}{d t}+\nabla w_{1}, \quad \frac{d v}{d t}=\frac{d z}{d t}+\nabla w_{2} \\
\frac{d^{2} u}{d t^{2}}=\frac{d^{2} y}{d t^{2}}+\frac{\partial^{2} w_{1}}{\partial t^{2}}, \quad \frac{d^{2} v}{d t^{2}}=\frac{d^{2} z}{d t^{2}}+\frac{\partial^{2} w_{2}}{\partial t^{2}}
\end{gathered}
$$

with

$$
\begin{aligned}
\nabla w_{k} \equiv & \frac{\partial w_{k}}{\partial t}+\frac{\partial w_{k}}{\partial \dot{y}} \frac{d^{2} y}{d t^{2}}+\frac{\partial w_{k}}{\partial \dot{z}} \frac{d^{2} z}{d t^{2}} \\
& +\frac{\partial w_{k}}{\partial y} \frac{d y}{d t}+\frac{\partial w_{k}}{\partial z} \frac{d z}{d t} ; k=1,2 .
\end{aligned}
$$

There are other terms in the partial derivative involving $((d y / d t),(d z / d t))$ as well as cross deriva- tives but they will make no contribution in the second averaging process here. Substitution into Eqs. (11) and grouping terms give

$$
\begin{aligned}
\frac{d^{2} y}{d t^{2}}= & c_{11} \frac{d y}{d t}+c_{12} \frac{d z}{d t} \\
& +c_{13} y+c_{14} z+g_{11}(t) \nabla w_{1} \\
& +g_{12}(t) \nabla w 2+g_{13}(t) w_{1}+g_{14}(t) w_{2} \\
& +U\left(w_{1,2}, \nabla w_{1,2}, y, z, \frac{d y}{d t}, \frac{d z}{d t}\right) \\
\frac{d^{2} z}{d t^{2}}= & c_{21} \frac{d y}{d t}+c_{22} \frac{d z}{d t} \\
& +c_{23} y+c_{24} z+g_{21}(t) \nabla w_{1} \\
& +g_{22}(t) \nabla w_{2}+g_{23} w_{1}+g_{24} w 2 \\
& +V\left(w_{1,2} \nabla w_{1,2}, y, z, \frac{d y}{d t}, \frac{d z}{d t}\right)
\end{aligned}
$$

where the second time derivatives of $w_{1,2}$ are balanced by the remaining terms according to

$$
\begin{aligned}
& \frac{\partial^{2} w_{1}}{\partial t^{2}}=\frac{C_{0}}{2} \frac{\partial w_{1}}{\partial t}+\frac{\omega}{2} \frac{\partial w_{2}}{\partial t}+G_{1} \\
& \frac{\partial^{2} w_{2}}{\partial t^{2}}=-\frac{\omega}{2} \frac{\partial w_{1}}{d t}+\frac{C_{0}}{2} \frac{\partial w_{2}}{d t}+G_{2}
\end{aligned}
$$


with

$$
\begin{aligned}
G_{k}= & g_{k 1} \frac{d y}{d t}+g_{k 2} \frac{d z}{d t}+g_{k 3} y \\
& +g_{k 4} z+H_{k}\left(\frac{\omega t}{2}\right) ; \quad k=1,2 .
\end{aligned}
$$

The functions $H_{k}$ are linear combinations of quadratic terms multiplied by trigonometric functions $[\cos (n \omega t / 2), \sin (n \omega t / 2) ; n=1,3]$. Through their appearance in the solutions to Eq. (15) they will combine with the same time dependencies in $U$, $V$ in Eq. (14) such that in the subsequent averaging process these functions quadratic in the coordinates now survive. On the other hand, to predict the onset of period two bifurcation, they do not have to be known explicitly. Stable asymptotic solutions $\partial w_{1,2} / \partial t$ are simply time integrals over $G_{1,2}$. Integrating these solutions with respect to time gives the result

$$
\begin{gathered}
w_{1}=A \sin (\omega t)-B \cos (\omega t) \\
w_{2}=B \sin (\omega t)+A \cos (\omega t) \\
A=\alpha_{11} \frac{d y}{d t}+\alpha_{12} \frac{d z}{d t}+\alpha_{13} y+\alpha_{16} z \\
B=\beta_{11} \frac{d y}{d t}+\beta_{12} \frac{d z}{d t}+\beta_{13} y+\beta_{14} z
\end{gathered}
$$

The constants $\alpha_{i j}, \beta_{i j}$ in terms of the system parameter are given in Table 2 . These constants are multiplied a common factor $\left[1 /\left(C_{0}^{2}+k \omega^{2}\right), k=1\right]$. Neglected are terms multiplied by a similar factor with $k=9$, consistent with the approximations limiting the period one orbit to be approximated by a simple harmonic oscillation. Since the generating functions of Eq. (16) are also of a simple harmonic form, $\overline{w_{k}}=0 ; k=1,2$. There are other terms linear in $\left[\nabla w_{k}, w_{k}\right]$ multiplying the coordinates, which, however, average to zero because of this property. It should be noted, on the other hand, that such terms could in principle have been included in Eq. (15) defining the generators prior to averaging. Also neglected are terms quadratic in $w_{k}$ therefore preserving the linearity of the second transformation Eq. (13). The approximations here are based on the principle of maximum symmetry for the eigenvalues characterizing the solution for the generators, consistent with the symmetry inherent in the defining linear van der Pol transformation equations (8). The result is that the generator solutions (16) display the same symmetry. Upon their insertion into Eq. (14), then with the use of Eq. (13), carrying out the averaging procedure results in the following coupled nonlinear differential equation system

$$
\begin{gathered}
\left(1-f_{11}\right) \frac{d^{2} y}{d t^{2}}-f_{12} \frac{d^{2} z}{d t^{2}}-d_{11} \frac{d y}{d t}-d_{12} \frac{d z}{d t} \\
=+e_{11} y+e_{12} z+\overline{U\left(y^{2}, y z, z^{2}, \ldots\right)} \\
-f_{21} \frac{d^{2} y}{d t^{2}}+\left(1-f_{22}\right) \frac{d^{2} z}{d t^{2}}-d_{21} \frac{d y}{d t}-d_{22} \frac{d z}{d t} \\
=+e_{21} y+e_{22} z+\overline{V\left(y^{2}, y z, z^{2}, \ldots\right)}
\end{gathered}
$$

where the coefficients $f_{i j}, d_{i j}, e_{i j}$ in terms of the system parameters are given in Table 3 . There are three formal differences between these renormalized equations averaged over the second transformation of Eq. (13) and the first averaged equations for which, from Eq. (11), $A_{i j}=c_{i j}, \overline{U(u, v)}=$ $\overline{V(u, v)}=0$. First, there are now nonzero quadratic terms in the transformed coordinates $y, z$ which function to define the existence of the period two orbit. Second, there are second derivative terms parameterized by $f_{i j}$, which do not appear in twodimensional studies [Holmes \& Holmes, 1981], but which function here to guarantee the stability of the period two orbit and fine tune the condition for the range of its stability. They were not included in the original study, for in that relatively simple case

Table 2. Coefficients $\alpha_{i j}, \beta_{i j}$ of Eq. (16).

These coefficients are given in terms of $a_{i j}, b_{i j}$ which are given explicitly in terms of the system parameters in Table $1 \alpha_{i j}=k_{1, j} / \omega, \beta_{i j}=k_{2, j} / \omega, \Gamma=C_{0}^{2}+\omega^{2}$, where

$$
\begin{aligned}
& \Gamma k_{11}=C_{0}^{2} \quad \Gamma k_{12}=-\omega C_{0} \quad \Gamma k_{21}=-\omega C_{0} \quad \Gamma k_{22}=-C_{0}^{2} \\
& \Gamma k_{13}=-\omega\left(a_{23}+b_{13}\right)+C_{0}\left(b_{23}-a_{13}\right) \quad \Gamma k_{14}=-\omega\left(a_{24}+b_{14}\right)+C_{0}\left(b_{24}-a_{14}\right) \\
& \Gamma k_{23}=\omega\left(a_{13}-b_{13}\right)-C_{0}\left(a_{23}+b_{13}\right) \quad \Gamma k_{24}=\omega\left(a_{14}-b_{24}\right)-C_{0}\left(a_{24}+b_{14}\right)
\end{aligned}
$$


Table 3. Coefficients $f_{i j}, d_{i j}, e_{i j}$ in Eq. (17).

These coefficients are given in terms of $k_{i j}$ from Table 2 , and in terms of the coefficients $\left[a_{i j}, b_{i j}, c_{i j}\right]$, which in turn are expressed explicitly in terms of the system parameters in Table 1.

$$
\begin{gathered}
m_{1}=\frac{1}{2}\left(b_{13}+a_{14}\right) \quad m_{2}=\frac{1}{2}\left(b_{14}-a_{13}\right) \quad m_{3}=\frac{1}{2}\left(b_{23}+a_{24}\right) \quad m_{4}=\frac{1}{2}\left(b_{24}-a_{23}\right) \\
\Gamma f_{11}=-\frac{C_{0}^{2}}{2} \quad \Gamma f_{12}=-\frac{C_{0}^{3}}{2 \omega} \quad \Gamma f_{21}=\frac{C_{0}^{3}}{2 \omega} \quad \Gamma f_{22}=-\frac{C_{0}^{2}}{2} \\
d_{11}=\left(\frac{k_{23}}{\omega}-k_{11}\right) \frac{C_{0}}{2}+\frac{k_{11} m_{1}}{\omega}+\frac{k_{21} m_{2}}{\omega}+c_{11} \quad d_{12}=+\left(\frac{k_{24}}{\omega}-k_{12}\right) \frac{C_{0}}{2}+\frac{k_{12} m_{1}}{\omega}+\frac{k_{22} m_{2}}{\omega}+c_{12} \\
d_{21}=\left(\frac{k_{13}}{\omega}+k_{21}\right) \frac{C_{0}}{2}+\frac{k_{11} m_{3}}{\omega}+\frac{k_{22} m_{4}}{\omega}+c_{21} \quad d_{22}=\left(\frac{k_{14}}{\omega}+k_{22}\right) \frac{C_{0}}{2}+\frac{k_{12} m_{3}}{\omega}+\frac{k_{22} m_{4}}{\omega}+c_{22} \\
e_{11}=-k_{13} \frac{C_{0}}{2}+\frac{k_{13}}{\omega} m_{1}+\frac{k_{23}}{\omega} m_{2}+c_{13} \quad e_{12}=-k_{14} \frac{C_{0}}{2}+\frac{k_{14}}{\omega} m_{1}+\frac{k_{24}}{\omega} m_{2}+c_{14} \\
e_{21}=k_{23} \frac{C_{0}}{2}+\frac{k_{13}}{\omega} m_{3}+\frac{k_{23}}{\omega} m_{4}+c_{23} \quad e_{22}=k_{24} \frac{C_{0}}{2}+\frac{k_{14}}{\omega} m_{3}+\frac{k_{24}}{\omega} m_{4}+c_{24}
\end{gathered}
$$

their contribution to the onset of $1 \leftrightarrow 2$ bifurcation was small. This is not so, however, in the more general case. Finally, the terms multiplying the coordinates, $e_{i j}$ and those multiplying the first derivatives, $d_{i j}$ are drastic modifications of their precursors $c_{i, j}$. They serve the pivotal function of determining the points in the control parameter space at which bifurcation occurs.

\section{The Act of Bifurcation: Creation and Stability of the Period Two Orbit}

The definition of the van der Pol transformation to coordinates $u, v$ of Eq. (8) combined with the subsequent transformation to the coordinates $y, z$ according to Eq. (13) implies that vicinal to the onset of periods $1 \rightarrow 2$ bifurcation the contribution to the period two orbit of Eq. (8a) is given approximately by $x_{2}=u_{o} \cos (\omega t / 2)-v_{o} \sin (\omega t / 2), u_{o} \simeq y_{o}$, $v_{o} \simeq z_{o}$ where $y_{o}, z_{o}$ are fixed points of Eq. (17),

$$
\begin{gathered}
e_{11} y_{o}+e_{12} z_{o}+U_{o}=0, \quad e_{21} y_{o}+e_{22} z_{o}+V_{o}=0 \\
\text { with } \quad U_{o}=\overline{U\left(y_{o}, z_{o}\right)} \quad \text { and } \quad V_{o}=\overline{V\left(y_{o}, z_{o}\right)}
\end{gathered}
$$

The range of stability of the period two orbit is that the parameter range within which the solution is stable as determined by the eigenvalue structure of Eqs. (17) linearized around the fixed point. Set- ting $y=y_{o}+y^{\prime}, z=z_{o}+z^{\prime}$ results in the following linearized equations,

$$
\begin{aligned}
& \left(1-f_{11}\right) \frac{d^{2} y^{\prime}}{d t^{2}}-f_{12} \frac{d^{2} z^{\prime}}{d t^{2}}-c_{11} \frac{d y^{\prime}}{d t}-c_{12} \frac{d z^{\prime}}{d t} \\
& =e_{11}^{\prime} y^{\prime}+e_{12}^{\prime} z^{\prime} \\
& -f_{21} \frac{d^{2} y^{\prime}}{d t^{2}}+\left(1-f_{22}\right) \frac{d^{2} z^{\prime}}{d t^{2}}+c_{21} \frac{d y^{\prime}}{d t}+c_{22} \frac{d z^{\prime}}{d t} \\
& =e_{21}^{\prime} y^{\prime}+e_{22}^{\prime} z^{\prime}
\end{aligned}
$$

where

$$
\begin{gathered}
e_{i j}^{\prime}=e_{i j}+\gamma_{i j} \\
\gamma_{11}=\left.\frac{\partial \bar{U}}{\partial y}\right|_{y_{o}, z_{o}} \quad \gamma_{12}=\left.\frac{\partial \bar{U}}{\partial z}\right|_{y_{o}, z_{o}} \\
\gamma_{21}=\left.\frac{\partial \bar{V}}{\partial y}\right|_{y_{o}, z_{o}} \quad \gamma_{22}=\left.\frac{\partial \bar{V}}{\partial z}\right|_{y_{o}, z_{o}}
\end{gathered}
$$

Solutions of the form $e^{\sigma t}$ upon substitution into Eq. (18a) results in the following eigenvalue equation

$$
\begin{aligned}
A_{4} \sigma^{4}+ & A_{3} \sigma^{3}+A_{2} \sigma^{2}+A_{1} \sigma+A_{0}=0 \\
A_{4}= & \left(1-f_{11}\right)\left(1-f_{22}\right)-f_{12} f_{21} \\
A_{3}= & -\left(d_{11}+d_{22}\right)+\left(d_{11} f_{22}+d_{22} f_{11}\right. \\
& \left.-d_{12} f_{21}-d_{21} f_{12}\right)
\end{aligned}
$$




$$
\begin{aligned}
A_{2}= & {\left[\left(d_{11} d_{22}-d_{12} d_{21}\right)-\left(e_{11}^{\prime}+e_{22}^{\prime}\right)\right] } \\
& +\left(e_{11}^{\prime} f_{22}+e_{22}^{\prime} f_{11}-e_{21}^{\prime} f_{12}-e_{12}^{\prime} f_{21}\right) \\
A_{1}= & \left(d_{11} e_{22}^{\prime}+d_{22} e_{11}^{\prime}\right)-\left(d_{12} e_{21}^{\prime}+d_{21} e_{12}^{\prime}\right) \\
A_{0}= & e_{11}^{\prime} e_{22}^{\prime}-e_{12}^{\prime} e_{21}^{\prime}
\end{aligned}
$$

This equation determines the conditions for stability of the period two orbit evolving from the period one orbit, parallel to the secular equation which determines the conditions for the evolution to a dynamical orbit, evolving from the instability of a fixed point. From the definition of $e_{i j}^{\prime}$ in Eq. (19) and expression of $e_{12}, e_{21}$ in terms of $e_{11}, e_{22}$ from the fixed point relations in Eq. (18), $A_{0}$ is given as

$$
\begin{aligned}
A_{0}= & -\left(\frac{e_{11} V_{o}}{z_{o}}+\frac{e_{22} U_{o}}{y_{o}}+\frac{U_{o} V_{o}}{y_{o} z_{o}}\right) \\
& +\left(e_{11} \gamma_{22}+e_{22} \gamma_{11}-e_{12} \gamma_{21}-e_{21} \gamma_{12}\right) \\
& +\left(\gamma_{11} \gamma_{22}-\gamma_{12} \gamma_{21}\right)
\end{aligned}
$$

Since $U_{o}, V_{o}$ and the derivatives $\gamma_{i j}$ are respectively quadratic and linear in the coordinates $y_{o}$, $z_{o}$, then $A_{0}=c_{1} y_{o}+c_{2} z_{o}$. The onset of bifurcation is when these coordinates emerge from zero. It follows that the first analytic condition for the onset of period one to period two bifurcation is $A_{0}=0$, at which point one of the roots of the eigenvalue equation (20) passes through zero. For the subsequent development of the period two orbit there must exist one real negative root and a pair of conjugate roots with negative real part. As the period two orbit develops, $y_{o}, z_{o}$ grow such that the period two orbit is stable as long as all roots are negative or have negative real part. When any real part becomes positive, the period two orbit itself becomes unstable. The calculation of that parameter point at which the period two orbit becomes unstable requires explicit inclusion of the nonlinear terms in $U$, $V$, as has been carried out in the previous study of a simple prototypic system [Phillipson \& Schuster, 1998]. However, only the requirement that they be nonlinear is sufficient to establish the necessary analytic condition $A_{0}=0$ for the onset of period two. The control parameter $r$ at the $1 \rightarrow 2$ bifurcation point is denoted here by $r^{*}$ so that $A_{k}^{*} \equiv A_{k}\left(r^{*}\right)$ and $A_{4}^{*} \sigma^{3}+A_{3}^{*} \sigma^{2}+A_{2}^{*} \sigma+A_{1}^{*}=0$. The necessary and sufficient condition that all eigenvalues have negative real parts and therefore the bifurcation is stable is when $A_{2}^{*} A_{3}^{*} \geq A_{1}^{*} A_{4}^{*}$ [Jackson, 1991].

The quantities $\rho_{o} \equiv\left(y_{o}, z_{o}\right)$ determine the amplitude and phase of the period two contribution to the period two orbit according to Eq. (5), where $\rho_{o}$ is the fixed point of the averaged equations (17) and given by Eq. (18). In terms of the coefficients of the secular equation (20), the two conditions for the onset of $1 \rightarrow 2$ bifurcation are

$$
A_{0}\left(\rho_{o}\right)=0
$$

and

$$
\begin{array}{r}
\Delta\left(\rho_{o}\right) \equiv A_{2}\left(\rho_{o}\right) A_{3}\left(\rho_{o}\right)-A_{1}\left(\rho_{o}\right) A_{4}\left(\rho_{o}\right)>0 \\
\text { in the limit } \rho_{o} \rightarrow 0 .
\end{array}
$$

As $\rho_{o}$ grows there are now four roots to Eq. (20). The extent of the period two orbit is determined by that value $\rho_{o}^{\prime}$ such that one or more of the roots acquires a positive real part, at which point the period two orbit becomes unstable [Phillipson \& Schuster, 1998].

\section{Bifurcation Analysis of Specific Dynamical Systems}

The present development rests on three assumptions. First, averaging as applied to two-dimensional system assumes that the external force constitutes a perturbation. This is a condition of smallness that can be imposed on the external force. Here the force, the period one orbit, is internally generated, and hence its strength is determined by the magnitude of control parameters in the region of bifurcation. Second, the period one oscillation is assumed to be soft and hence represented as a simple harmonic form which is modified by the addition of a small period two contribution at the point of bifurcation. This condition of smallness is reflected by the construction Eq. (13) which represents the approximation of a smooth, linear nearidentity transformation. Finally, bifurcation plots indicating the onset of period two bifurcation occur symmetrically with respect to the period one orbit. On this basis the approximation was additionally made that the symmetry inherent to the van der Pol transformation Eq. (9) is preserved by the second transformation Eq. (13) through the symmetric form of the generators in Eq. (16). The prediction of the onset of instability of a fixed point is, in principle, known precisely through algebraic procedures by the determination of the fixed point in terms of the control parameters. These assumptions of the present work places restriction on quantitative prediction. The purpose of this section is to evaluate 
the severeness of this restriction by considering four examples.

\subsection{Rössler equations}

Setting $\mathbf{X}=(X, Y, Z)$ the following equations of Rössler [1976]

$$
\begin{aligned}
& \frac{d X}{d t}=-(Y+Z) \\
& \frac{d Y}{d t}=X+a Y \\
& \frac{d Z}{d t}=b+X Z-r Z
\end{aligned}
$$

may be considered the most elementary construction of chaos in continuous systems [Peitgen et al., 1992]. This judgement is reinforced by the observation that by choosing $x=Y$, the transformation to the generalized quadratic form (4) is exact, with the parameters given by

$$
\begin{aligned}
g_{1}=-r & g_{2}=a r-1 \quad g_{3}=a-r \quad k=-b \\
q_{11}=-a & q_{12}=1+a^{2} \quad q_{13}=-a \\
q_{22} & =-a \quad q_{23}=1 \quad q_{33}=0
\end{aligned}
$$

Figure 1 shows a bifurcation diagram for the Rössler system: values of $Y=x$ coordinate, $x_{n}$, are plotted against $r$ regarded as a continuously varying control parameter for two representative cases, (a) $a=b=0.2$ and (b) $a=0.3, b=0.2$. In both plots $1(\mathrm{a})$ and $1(\mathrm{~b})$ the arrow indicates the locus of the $1 \rightarrow 2$ bifurcation, subsequent to which occur period doublings with increasing $r$, approaching chaos with very different scenarios: the first case exhibits "spiral-type chaos" and the second case "screw-type" chaos [Jackson, 1991]. Of present concern is the observation that the transition from case (a) to case (b) is also accompanied by a shift of the bifurcation point towards a smaller value. In general, for a given $(a, b)$ pair if $b \cong a, r^{*}(b>a)>r^{*}(b<a)$. It is possible to trace this analytically, since in the region of bifurcation, $a, b \ll r$ insertion of the parameters Eq. (24) into Eq. (6) gives the following approximate period one orbit through terms linear in $a$

$$
\begin{gathered}
x_{1}(t)=-a\left(r+\frac{1}{r}\right)+\left[2\left(r^{2}+\frac{a-b}{a}\right)\right]^{\frac{1}{2}} \cos (\omega t), \\
\omega=\left(1+\frac{a}{r}\right)^{\frac{1}{2}}
\end{gathered}
$$

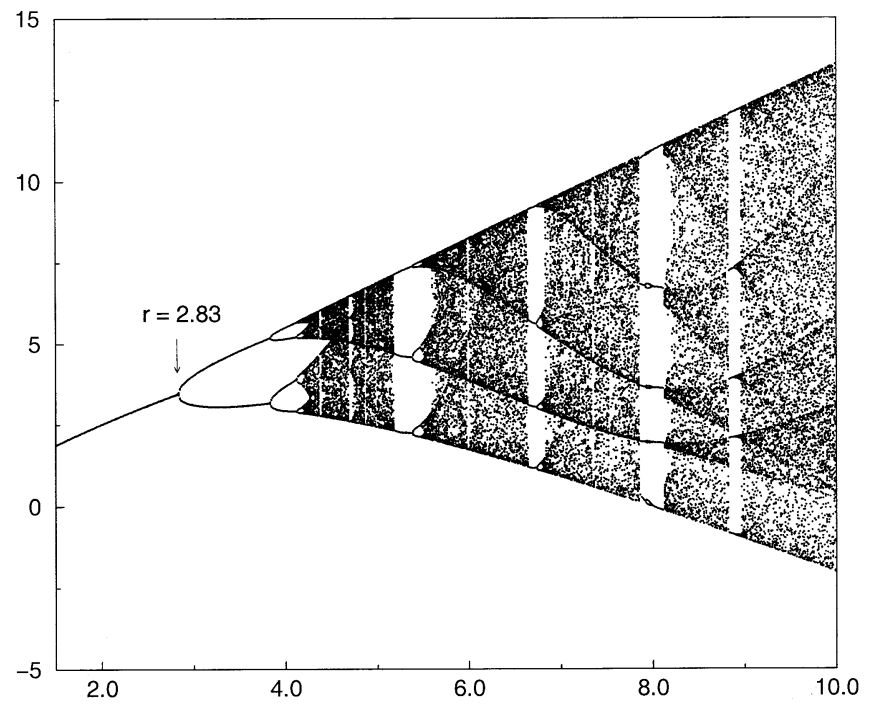

(a)

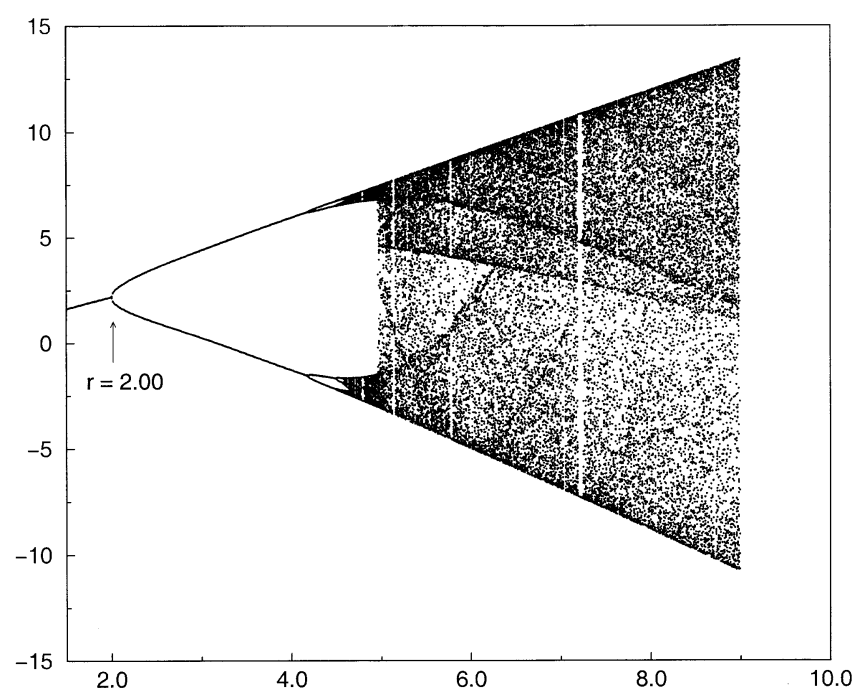

(b)

Fig. 1. Bifurcation diagram $x_{n}$ versus $r$ generated by computer solution of the Rössler equations (23) computed by the exact transformed equations (4) with the parameters given in Eq. (24). The points $x_{n}$ are the values of $x$ recorded at those times $t=t_{n}$ at which this coordinate reaches a maximum. The arrow indicates the location of the $1 \rightarrow 2$ bifurcation at $r^{*}$ (a) $a=b=0.2, r^{*}=2.83$ (b) $a=0.3 b=0.2, r^{*}=2.00$.

Equations (24) and (25) complete the necessary parameter specification to satisfy the conditions for Eq. (22). The results are: Case (a) $r^{*}=2.97$ [2.83], Case (b) $r^{*}=1.78$ [2.00] where the numbers in brackets are the $1 \rightarrow 2$ bifurcation points determined by computer solution of (23). A typical trend, for example is when $a=0.3$ and $b=0.2$, $0.3,0.4$ the points of bifurcation are 1.78 [2.00], 1.94 
[2.10], 2.09 [2.25], respectively. These results indicate that the present approximations are satisfactory for predicting the $1 \rightarrow 2$ bifurcation points as a function of control parameters within $10 \%$ accuracy, and that the error is consistently in the direction of values too small by this factor. The primary reason for this quantitative discrepancy is the restriction of the period one orbit to a simple harmonic form.

\subsection{Lorenz equations}

Setting $\mathbf{X}=(X, Y, Z)$ and $\varepsilon \equiv 1 / \sqrt{r}$ the Lorenz equations [Lorenz, 1963]

$$
\begin{gathered}
\frac{d X}{d t}=Y-\varepsilon \sigma X \\
\frac{d Y}{d t}=(\sigma X-X Z)-\varepsilon Y \\
\frac{d Z}{d t}=X Y-b \varepsilon Z \\
{\left[X \rightarrow \frac{X}{\varepsilon}, Y \rightarrow \frac{Y}{\sigma \varepsilon^{2}}, Z \rightarrow \frac{Z}{\sigma \varepsilon^{2}}, t \rightarrow \varepsilon t,\right.} \\
\text { and } \quad K \equiv \frac{X^{2}}{2}-Z \quad \text { yield } \\
\left.\frac{d K}{d t}=-2 \varepsilon \sigma K-\varepsilon(2 \sigma-b) Z,\right]
\end{gathered}
$$

with $\sigma=10, b=8 / 3$ and $r$ as a control parameter, demonstrated for the first time that deterministic chaos could arise in a three-dimensional autonomous differential equation system. When $r \cong 28(\varepsilon \cong 0.189)$ these equations demonstrate chaotic oscillations of a strange attractor. As $r$ increases there exist sequences of periodic oscillations punctuated by aperiodic and chaotic regions, finally terminating with a $2 \rightarrow 1$ bifurcation at $r_{2 \rightarrow 1}=313$ $(\varepsilon=0.0565)$. These sequences are shown in the bifurcation diagram for $Z$ in Fig. 2(a) computed from Eq. (26a). The present study is concerned with this bifurcation in the region of relatively large $r(\varepsilon \ll 1)$ so that the original equations have been scaled according to the prescription of Sparrow [1982]. The relation of Eq. (26b), a consequence of the Lorenz equations, will prove useful in the analysis.

Transformation of the Lorenz equations to a single third-order differential equation of the form Eq. (3a) choosing $x \equiv Z$ leads to the following exact but complicated result derived in the Appendix

$$
\begin{aligned}
& W(x, y, z) \\
& =-\varepsilon(b+\sigma+3) z-\varepsilon^{2}[b(\sigma+3)+2(\sigma+1)] y \\
& -2 b \varepsilon^{3}(\sigma+1) x+4(\sigma-x)(y+b \varepsilon x) \\
& -[2 \varepsilon(\sigma-1)(\sigma-x)+y] X^{2}(x, y, z) \\
& X^{2}(x, y, z)=\frac{B-\sqrt{B^{2}+4(x-\sigma) A}}{2(\sigma-x)} \\
& A=(y+b \varepsilon x)^{2} \\
& B=z+\varepsilon(b+\sigma+1) y+b \varepsilon^{2}(\sigma+1) x
\end{aligned}
$$

Computation of the bifurcation diagram from large to small values of $r$ produces the identical result in Fig. 2(a) until $r=198$ where the attractor is beginning to expand. At that point the square root in the expression for $X^{2}$ becomes imaginary and the transformation becomes improper for $r \geq 198$. A study of the Lorenz equations [Michielin \& Phillipson, 1997] developed a one-dimensional mapping which for small $r$ behaves as a tent map (whose iterates are chaotic) and for large $r$ behaves as a quadratic map (whose iterates are universal [Metropolis et al., 1973]). The result was prediction of the ordering of periodic orbits over the range of control parameter. It was found that a transitional value $r_{t}=193.126$ is such that for $r>r_{t}$ iterates are confined to a map region characterized by the quadratic portion of the mapping and for $r<r_{t}$ the iterates escape this region and ultimately condense to the chaotic structure initiated around $r=28$. This value of $r_{t}=193$, found from an approximate map construction, is sufficiently close to the point 198 obtained by numerical integration of the exact equations, to identify these points. The transformation is valid as long as all orbits are confined to a sequence which correlates essentially with the universal period doubling sequence. It fails abruptly as the sequence of orbits approaches chaos with an ordering specific to the Lorenz equations.

Since the inverse $2 \rightarrow 1$ bifurcation of interest is far from $r_{t}$ we will first dismantle the attractor by setting $\varepsilon=0$ and then approximately reconstruct it for $\varepsilon \ll 1$. Sparrow [1982] has dealt in some detail with analysis of the Lorenz equations for the case of small $\varepsilon$, showing how exact solutions for $\varepsilon=0$ can be expressed in terms of elliptic integrals and approximate but complicated solutions generated for $\varepsilon$ small using averaging techniques. The present 


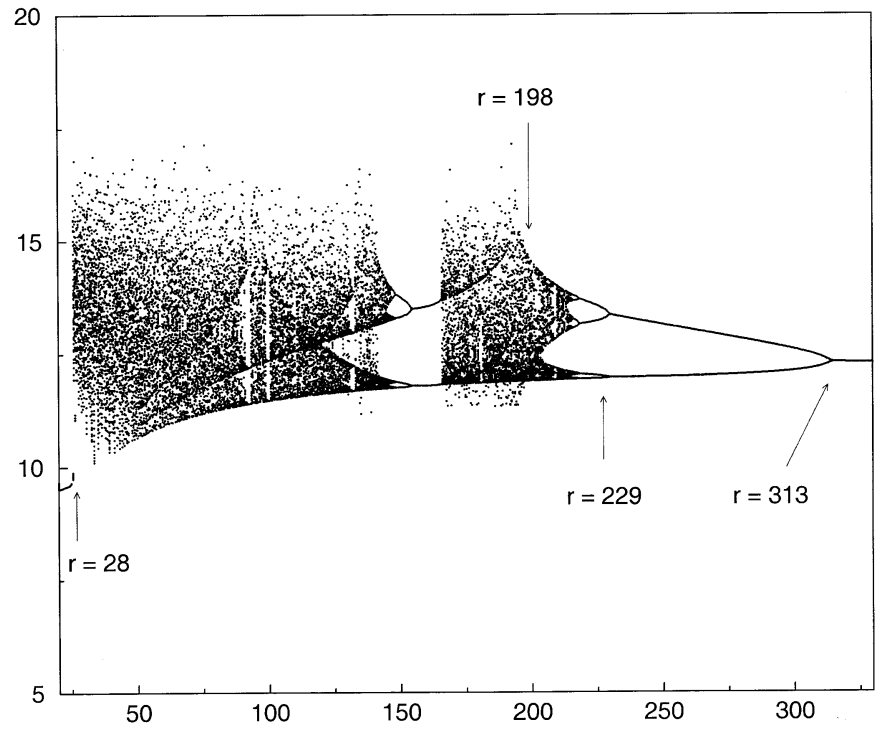

(a)

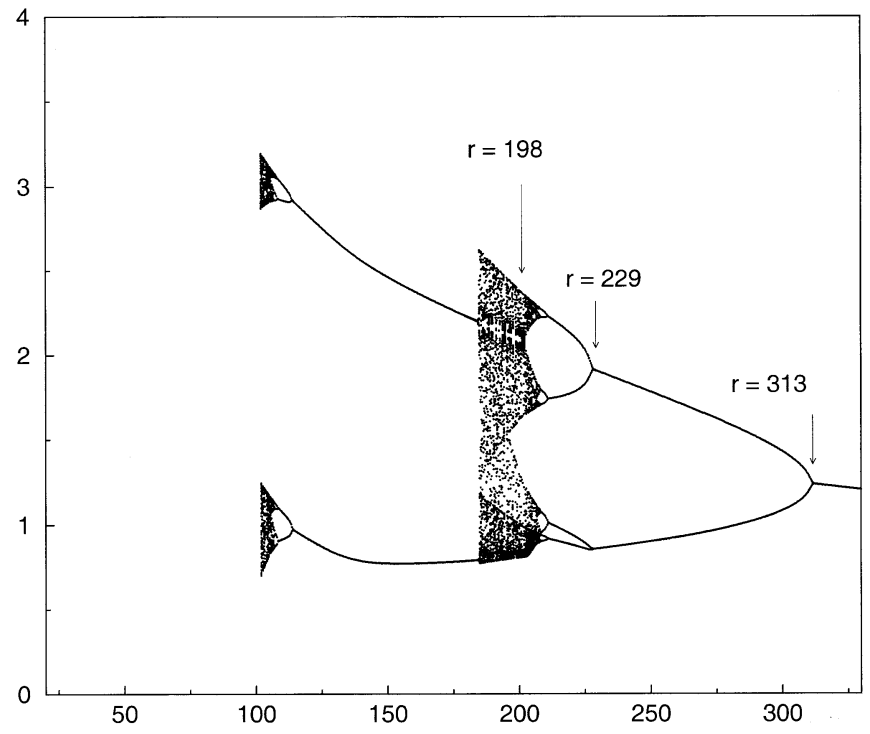

(b)

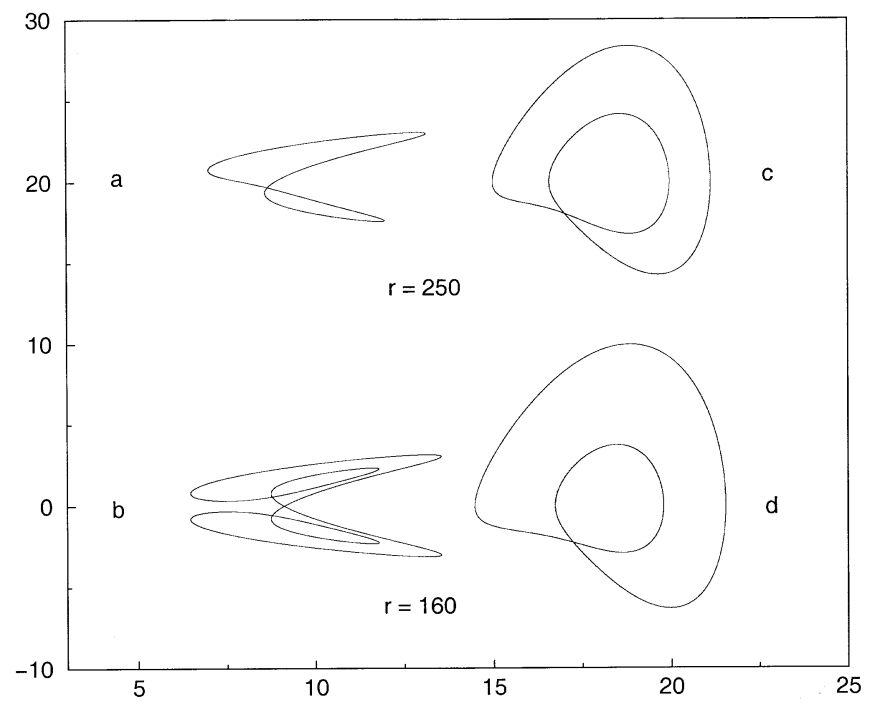

(c)

Fig. 2. The Lorenz equations. (a) Bifurcation diagram $Z_{n}$ versus $r$ generated by the computer solution of the Lorenz equations (26a) where the points are the oscillation maxima as in Fig. 1. The arrows indicate significant values of $r$ in the order of increasing $r$ : [28] transition to chaos originally reported by Lorenz [1963], [198] point at which the transformed Lorenz equations becomes ill-defined, indicating an abrupt transition in the dynamics, [229] $4 \rightarrow 2$ bifurcation point, [313] $2 \rightarrow 1$ symmetry breaking bifurcation point. (b) Bifurcation diagram of reconstructed attractor Eq. (4) with parameters of Eq. (30). This attractor ends at $r=102$. (c) Phase plots for the Lorenz equations computed from Eq. (26a) for $r=250$ in the region of coexisting single cycles and $r=160$ in the region of a period two orbit. The figures (a) and (b) on the left are plots of $X$ versus $Z$ whose difference reflects the fact that the former is the result of a symmetry breaking bifurcation and the latter is the result of period doubling. The figures (c) and (d) on the right are the phase plots $y=[d Z / d t]$ versus $x=Z$ whose similarity reflects that in these dynamical coordinates both cases appear as a $1 \rightarrow 2$ bifurcation. 
scheme starts from the third-order equation (27) and invokes a computer-assisted approach to produce a rather simple equation which reproduces the dynamics for large $r$ in reasonable detail. Formally Eq. (26b) can be integrated

$$
\begin{aligned}
X_{\varepsilon}^{2}= & 2 K_{o} e^{-2 \varepsilon \sigma t} \\
& +2\left[x-\varepsilon(2 \sigma-b) \int_{0}^{t} e^{-2 \varepsilon \sigma(t-t \prime)} x\left(t^{\prime}\right) d t^{\prime}\right] .
\end{aligned}
$$

When $\varepsilon=0$, there is a unique symmetric conservative periodic orbit characterized by two constants of the motion, one of which is $K_{o}$, where $X_{o}^{2}=2\left(x+K_{o}\right)$. From Eq. (27), when $\varepsilon=0$, $X^{2}=0$ at $y=0$. This corresponds to the minimum of the oscillation, $x=x_{\text {min }}$, so that $X_{o}^{2}=$ $2\left(x-x_{\min }\right), K_{o}=-x_{\min }$ and the Lorenz equations can be written as a third-order differential equation according to $d z / d t=\left[\left(2 x_{\min }+4 \sigma\right)-6 x\right] y$. The oscillation is conservative and the unique solution requires specification of two initial conditions $\left[x_{\min }, z_{\min }\right]$ the family of which solutions lie on a cylinder. This is equivalent to expressing $\varepsilon=0$ equation as a first-order differential equation where both constants would appear explicitly and the solution is expressed in terms of elliptic integrals. For $\varepsilon>0$ the first (now transient) term asymptotically goes to zero, only the integral remains and the oscillation is a limit cycle. It is further assumed that the oscillation in the period one region has the generic structure $x\left(t^{\prime}\right)=k(\varepsilon)+u\left(t^{\prime}\right), k(\varepsilon)=k_{0}+k_{1} \varepsilon$ where $u\left(t^{\prime}\right)$ is an oscillatory function which will be neglected, so that $X_{\varepsilon}^{2} \cong 2[x-(1 / 2)(1-(b / \sigma)) k(\varepsilon)]$. Retaining terms only linear in $\varepsilon$ the Lorenz equations in the form of Eq. (27) are approximated by

$$
\begin{gathered}
\frac{d z}{d t}=- \\
+[2 \varepsilon(b+\sigma+3) z+4(\sigma-x)(y+b \varepsilon x) \\
X_{\varepsilon}^{2}(x)=2\left(x-x_{\min }\right)-\gamma \varepsilon
\end{gathered}
$$

where the constants $k_{0,1}$ have been redefined in terms of constants $x_{\min }, \gamma$. Consistent with the fact that the Lorenz equations have a fixed point $x_{o}=\sigma\left(1-\varepsilon^{2}\right) \cong \sigma, \varepsilon>0$ then with respect to this point, Eq. (29) is of the quadratic form Eq. (4) with

$$
\begin{gathered}
g_{1}=-2 \varepsilon\left[2 b \sigma-(\sigma-1) X_{\varepsilon}^{2}(\sigma)\right] \\
g_{2}=-X_{\varepsilon}^{2}(\sigma) \quad g_{3}=-\varepsilon(b+\sigma+3) \\
q_{11}=4 \varepsilon(\sigma-b-1) \quad q_{12}=-6
\end{gathered}
$$

Figure 2(b) shows the bifurcation diagram for the Lorenz equations reconstructed according to Eq. (4) with parameters of Eq. (29) and $\left[x_{\min }=8.262, \gamma=\right.$ 24.96]. These values were determined by matching the bifurcation diagram to the computed $2 \rightarrow 1$, $4 \rightarrow 2$ bifurcation points at $r=313$ and $r=229$ respectively. Comparison with Fig. 2(a) indicates that this quadratic reconstruction is sufficient to reproduce the Lorenz attractor in the period doubling region with reasonable fidelity. On the other hand, a separate computer study of Lorenz equations (26) in the range $300<r<10^{6}$ showed numerically as $\varepsilon \rightarrow 0$, the extrapolated values $x_{\min }=8.456$, $z_{\min }=1.518$. The first figure is larger than the one adopted for the present simple two parameter fit to the attractor, 8.262 , by approximately $3 \%$. It should also be noted, from Eq. (27), that an expansion of $X^{2}$ in powers of $\varepsilon$ is not analytic at $x=\sigma, z=0$ because there is no fixed point when $\varepsilon=0$. This discontinuity reflects the fact that in this region of two basins of attraction the bifurcation is a symmetry breaking bifurcation, not a normal pitchfork period doubling bifurcation, although the two prongs emerging from the transition point appear normal [Froyland \& Alfsen, 1984]. When $\varepsilon=0$ the conservative orbit is invariant under $(X, Y, Z) \rightarrow(-X,-Y, Z)$ which is preserved up to the bifurcation point where it becomes unstable and replaced with two stable limit cycles of opposite symmetry $( \pm X, \pm Y, Z)$. These two orbits are the two prongs. The phase plots of Fig. 2(c) indicate the differences between the symmetry breaking bifurcation in the two single cycle region at $r=250$ to be compared with the period doubling orbit at $r=160$. The phase plots of $d Z / d t$ versus $Z$ (ordinate and abscissa axes, respectively) show essentially the same double loop characteristic of a period doubled orbit, while the phase plots $X$ versus $Z$ betray (for a given choice of initial conditions) a single cycle structure at $r=250$ and a two cycle structure at $r=160$ (for any initial condition). For this reason, and as noted by Froyland and Alfsen, the bifurcation plot does not distinguish between orbits of different symmetry.

The parameters of the period one orbit, from Eqs. (4) and (30) are: $D_{0}=(4 \sigma b-\gamma(3 \sigma+b+$ $1)) /(14 \sigma-2 b+10), D_{1}^{2}=D_{0}[[(2 b \sigma-(\sigma-1) \gamma) /(\sigma-$ $\left.b-1)]-2 D_{0}\right], \omega^{2}=\gamma+6 D_{0}$. With the parameters $x_{\min }=8.262, \gamma=24.96$ adjusted to reproduce the attractor, the second condition of Eq. (22) is not satisfied. This discrepancy might not be unexpected in view of the approximation of the exact 
Lorenz attractor by Eq. (30). Nevertheless, it is quite small, for when $x_{\min }$ is reduced by less than $1 \%$ to $x_{\min }=8.236$ both conditions are satisfied at a bifurcation point 315 which compares quite favorably with the computer value of 313 . This indicates on the one hand the extreme sensitivity of the precise location of the bifurcation point to parameter values and, on the other hand, that subject to this limitation the present asymptotic method provides a picture of the mechanism of bifurcation.

\subsection{Replicator equations}

Catalyzed replication and mutation find the most simple expression by the following mechanism which has been implemented in a flow reactor [Schuster et al., 1980; Hofbauer \& Sigmund, 1988]: $(S)+I_{j}+I_{i} \rightarrow I_{j}+I_{k}+I_{i}, i, j, k=1,2, \ldots, n$ where $I_{j}$ is the template which is replicated, $I_{i}$ the catalyst and $(S)$ are the buffered substrates needed for replication. These equations describe a kinetic mechanism whereby $I_{i}$ catalyzes the replication of $I_{j}$ which yields $I_{k}$ being either a correct copy $(k=i)$ or an error copy called mutant $(k \neq i)$. The system is closed in that the conservation of mass leads to $\sum_{i=1}^{n}\left[I_{i}\right]=c_{o}=$ const. and this restriction implies for an $n$ species system that there are only $n-1$ independent equations. For $n=4$ and a representative choice of replication matrix the replicator equations under a Hofbauer transformation [Hofbauer, 1981] is identical to the following generalized Lotka-Volterra system studied by Arneodo et al. [1980]:

$$
\begin{aligned}
& \frac{d X}{d t}=X[0.5(1-X)+0.5(1-Y)+0.1(1-Z)] \\
& \frac{d Y}{d t}=Y[-0.5(1-X)-0.1(1-Y)+0.1(1-Z)] \\
& \frac{d Z}{d t}=Z[r(1-X)+0.1(1-Y)+0.1(1-Z)]
\end{aligned}
$$

The bifurcation diagram of $Z$ for this system shown in Fig. 3(a), first analyzed by Schnabl et al. [1991], shows that the attractor exhibits initially a period doubling scenario which very closely resembles that predicted by a quadratic map followed subsequently by an interior crises resulting in a period two tangent bifurcation, this process repeating until the end of the attractor. This extended scenario of periodic orbits embedded in chaotic regions has been analyzed in the context of one-dimensional and twodimensional mappings [Phillipson \& Schuster, 1994;
Michielin \& Phillipson, 1994]. Choosing $x$ such that $Z=e^{x}$ then the third-order differential equation would be a function of this exponential coordinate. Noting that the interior fixed point is at $[1,1,1]$, the quadratic form of Eq. (4) is achieved in the approximation of expanding the exponential terms through $x^{2}$ and neglecting higher terms. In this case, the quadratic form represents an expansion around the fixed point such that the linear terms provide the secular equation to predict its instability by Hopf bifurcation and the quadratic terms serve to encapsulate the subsequent period doubling dynamics. The coefficients are $h=0$ and

$$
\begin{aligned}
g_{1} & =-(0.06 r+0.01) \quad g_{2}=0.1 r-0.23 \\
g_{3} & =-0.5 \quad q_{11}=-0.003(6 r+1) \\
q_{12} & =0.1 r-0.014 \quad q_{13}=-0.24 \\
q_{22} & =-0.2 \quad q_{23}=\frac{0.04}{r} \quad q_{33}=-\frac{0.8}{r}
\end{aligned}
$$

The coefficients $q_{i j}$ involve the ratio of polynomials in $r$. Only the dominant term for each coefficient has been retained, which introduces an error in the $1 \rightarrow 2$ bifurcation region of interest quantitatively of less than $3 \%$. As distinct from the previous two cases, there is a $q_{33} z^{2}$ contribution without which calculation shows there is no bifurcation in the present approximation. Figure 3(b) shows the bifurcation diagram for this system. Up to the period three window the attractor presents a period doubling sequence which reproduces the exact attractor with good fidelity: the first two bifurcations occur at $1.28(1 \rightarrow 2), 1.34(2 \rightarrow 4)$ to be compared with the accurate values $1.30,1.37$ respectively. The subsequent severe distortion in the period three range and beyond is due in this approximation to the relatively large magnitude of $q_{13}$. Reduction of its value from 0.24 to 0.14 results in the bifurcation diagram of Fig. 3(c) which not only restores the proper position of these bifurcations but also now includes properly period two bifurcations of the extended attractor. This shows the limitations of the quadratic approximation, but also indicates that the approximation can be formally explored to produce more global characteristics of an attractor.

The analytical period one orbit is dependent ultimately on the behavior of $D_{0}$ in Eq. (6) which is seen to vanish when $g_{1}+g_{2} g_{3}=0$. Since the $g_{k}$ 's determine the eigenvalues for analysis of fixed point stability, this is the condition for a Hopf bifurcation. In the present case, from Eq. (32), this 


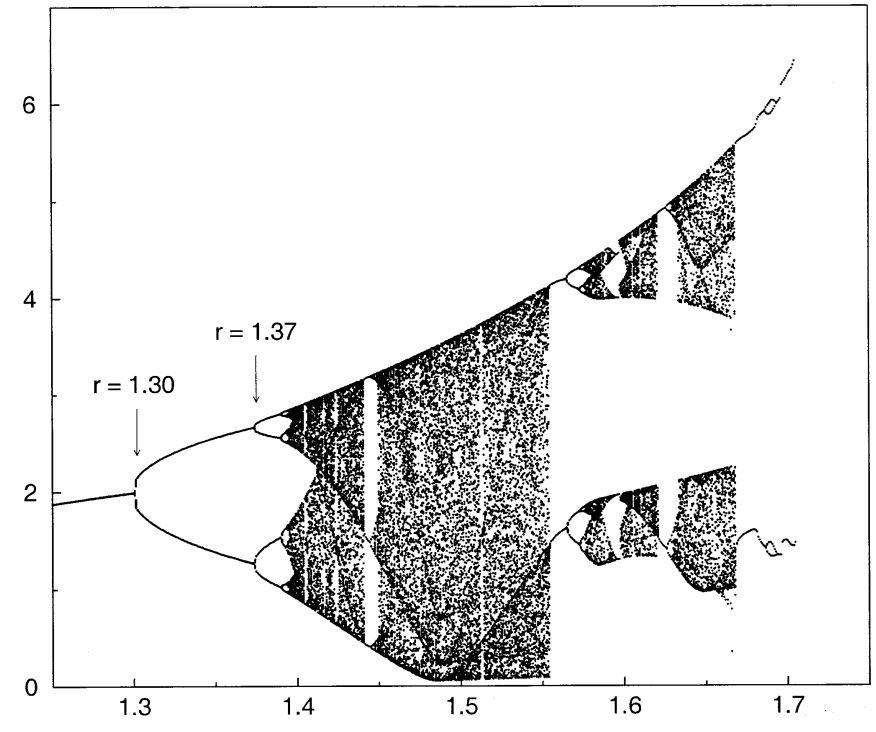

(a)

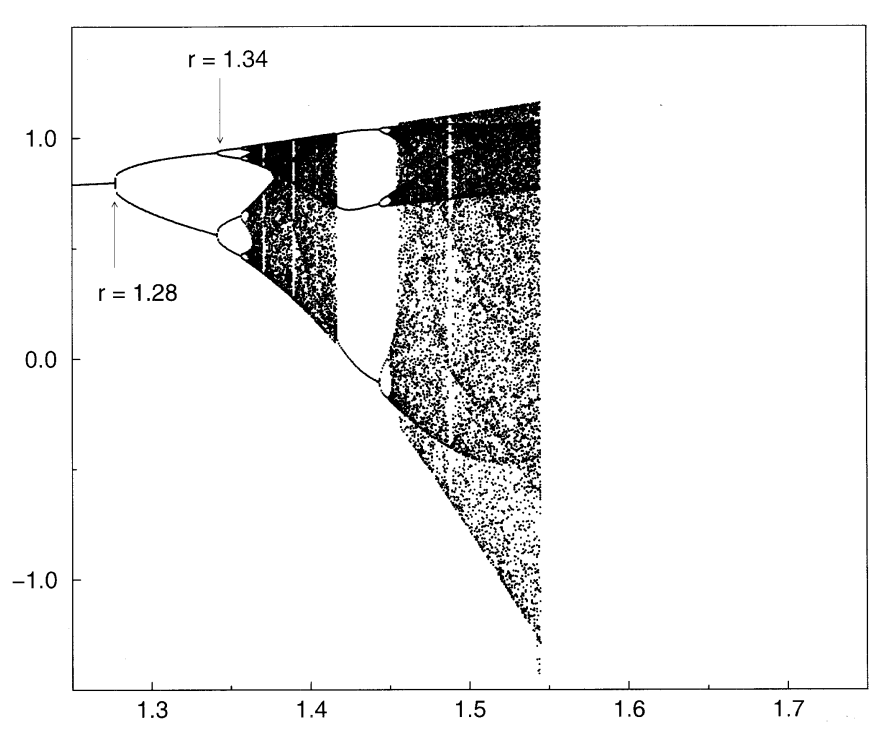

(b)

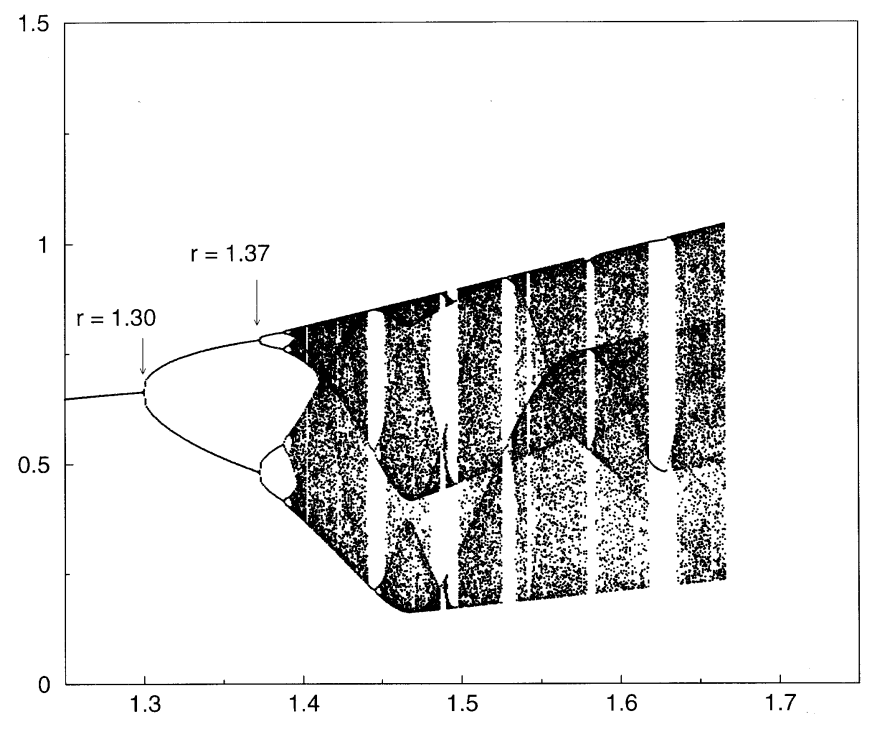

(c)

Fig. 3. Bifurcation diagrams for the three-dimensional replicator equation where the points are the oscillation maxima as in Fig. 1. (a) $z_{n}$ versus $r, z_{n}=Z_{n}-1$ generated by the computer solution of Eq. (31) where the values are with respect to the interior fixed point $[1,1,1]$. The arrows indicate the points of the $1 \rightarrow 2$ bifurcation at $r^{*}=1.30$ and the $2 \rightarrow 4$ bifurcation at $r=1.37$. (b) Replicator attractor constructed according to Eq. (4) with parameters of Eq. (32). (c) Replicator attractor constructed according to Eq. (4) with parameter of Eq. (32) except $q_{13}=-0.14$.

quantity is $-0.11\left(r-r_{o}\right), r_{o}=(10.5 / 11)=0.9545$. At $r_{o}$ the fixed point becomes unstable and the period one orbit grows until it becomes unstable when the conditions of Eq. (22) are satisfied. Calculation with the coefficients of Eq. (32) reveal two values of $r=1.28,1.32$, the first one of which agrees with Fig. 3(b). There is no reason in principle why there cannot be more than one value of the control parameter for a system which would satisfy these conditions. An example is the previous study [Phillipson $\&$ Schuster, 1998]. If such is the case, which one or ones are the correct candidates must be decided on the basis of additional theoretical or computational considerations. 


\subsection{Chua's circuit equations}

Chua's circuit is the simplest autonomous generator of chaotic signals [Chua, 1992] and as such has been the subject of extensive studies in recent years. Setting $\mathbf{X}=(X, Y, Z)$ the normalized state equations are [Khibnik et al., 1993]

$$
\begin{aligned}
& \frac{d X}{d t}=\alpha[Y-\phi(X)] \\
& \frac{d Y}{d t}=X-Y+Z \\
& \frac{d Z}{d t}=-\beta Y
\end{aligned}
$$

where $\alpha, \beta$ are control parameters and $\phi(X)$ is a nonlinear function. Transformation to the form in Eq. (3a) for the coordinate $X$ gives the result

$$
\begin{aligned}
\frac{d^{3} X}{d t^{3}}= & -\left\{\left[1+\alpha \frac{d \phi}{d X}\right] \frac{d^{2} X}{d t^{2}}+[(\beta-\alpha)\right. \\
& \left.\left.+\alpha \frac{d \phi}{d X}\right] \frac{d X}{d t}+\alpha \frac{d^{2} \phi}{d X^{2}}\left(\frac{d X}{d t}\right)^{2}+\alpha \beta \phi(X)\right\}
\end{aligned}
$$

Madan and $\mathrm{Wu}$ [1993] derived an equivalent thirdorder differential equation in $Z$ which is free of derivatives of $\phi$, choosing a discontinuous function of three linear time segments in $X(Z)$. However, the nonlinearity of a real circuit is smooth, prompting Khibnik et al. [1993] to adopt, as we will here, the following cubic nonlinearity

$$
\phi(X)=-d_{1} X+d_{3} X^{3} \quad d_{1}=\frac{1}{6} \quad d_{3}=\frac{1}{16}
$$

for which smoothness of the function presents no analytic difficulties involving derivatives of $\phi$. With respect to the fixed point, $\phi\left(x_{o}\right)=0,\left[x_{o}=\right.$ $\left.\left(d_{1} / d_{3}\right)^{\frac{1}{2}} \approx 1.633\right]$, setting $X=x_{o}+x$, Eq. (34) assumes the following explicit form of (3a) and (3b)

$$
\begin{gathered}
\frac{d z}{d t}=g_{1} x+g_{2} y+g_{3} z+Q(x, y, z)+C(x, y, z) \\
Q(x, y, z)=q_{1} x^{2}+q_{2}\left[x y+x z+y^{2}\right] \\
C(x, y, z)=c_{1} x^{3}+c_{2}\left[2 x y^{2}+x^{2} y+x^{2} z\right]
\end{gathered}
$$

where

$$
\begin{aligned}
& g_{1}=-2 \alpha \beta d_{1} \quad g_{2}=-\left[(\beta-\alpha)+2 \alpha d_{1}\right] \\
& g_{3}=-\left[1+2 \alpha d_{1}\right] \quad q_{1}=-3 \alpha \beta d_{3} x_{o} \\
& q_{2}=-6 \alpha d_{3} x_{o} \quad c_{1}=-\alpha \beta d_{3} \quad c_{2}=-3 \alpha d_{3}
\end{aligned}
$$

Figure 4(a) shows a bifurcation diagram $x_{n}$ versus $\alpha$ for the Chua circuit computed from these exact equations, (36) and (37) with $\beta=14$. It is similar but with minor differences to that shown by Madan and Wu [1993] for the discontinuous nonlinearity function. The arrows indicate the

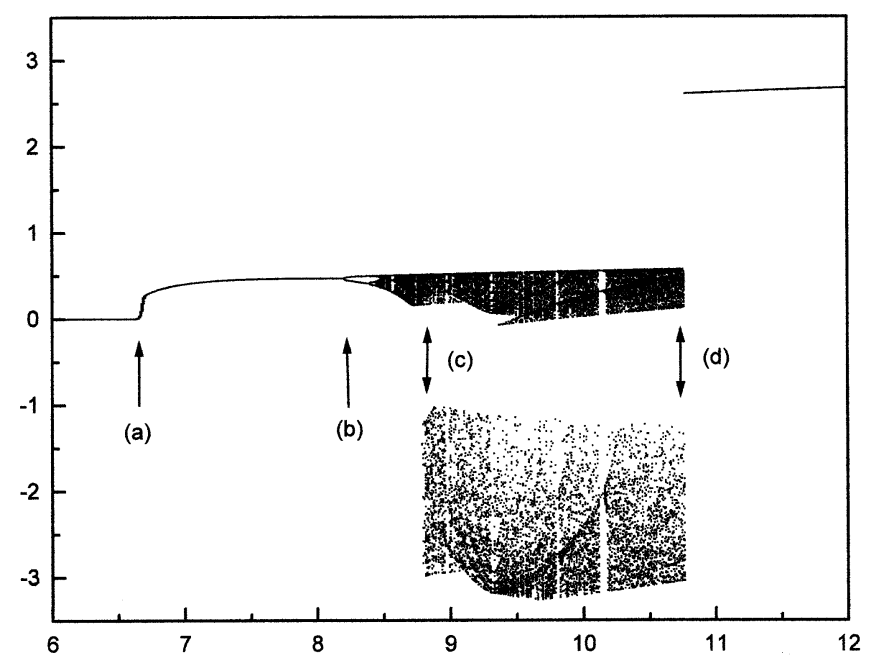

(a)

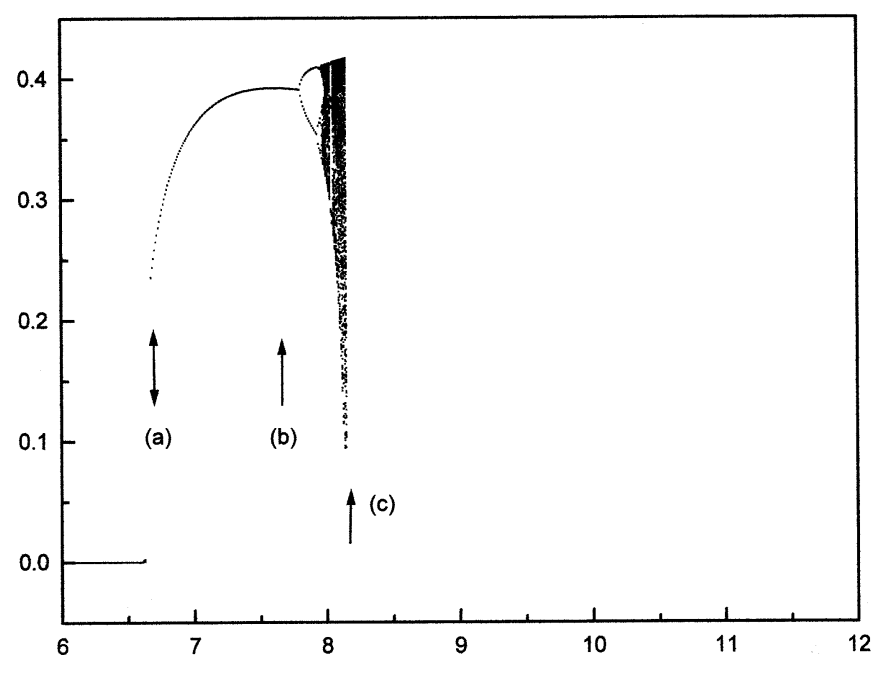

(b)

Fig. 4. Bifurcation diagrams $x_{n}$ versus $\alpha, \beta=14$ for Chua's circuit where the points are oscillation maxima as in Fig. 1. (a) Computer solution of exact equations (36) with parameters given in Eq. (37). The arrows indicate the progressive initiations of dynamical events discussed in the text. (b) Computer solution of Eq. (36) in the quadratic approximation of Eq. (4) $[C(x, y, z) \equiv 0]$. The $\alpha$-scale is the same as in Fig. 4(a) to indicate that the attractor breaks off approximately where the double-scroll attractor would begin. The arrows (a), (b) and (c) indicate the locations of Hopf bifurcation, $1 \rightarrow 2$ period doubling and the end of the attractor respectively. 
progression of the dynamics with increasing $\alpha$. This scenario, discussed in detail by Khibnik et al. [1993] indicates: (a) Hopf bifurcation at $\alpha=$ $\left.\left(\sqrt{1+\left[8 \beta d_{1} /\left(1-2 d_{1}\right)\right]}-1\right) / 4 d_{1}\right) \quad[6.578]$, then (b) $1 \rightarrow 2$ bifurcation at $[\alpha=8.198]$ followed by a period doubling cascade which terminates at (c) $[\alpha=8.785]$. Beyond this location the expansion of points indicates the birth of the Chua double-scroll attractor which persists until its disappearance at (d) $[\alpha=10.769]$. Beyond this point, the straight line indicates the system has regressed to a simple period one orbit. By contrast Fig. 4(b) shows the same bifurcation diagram in the quadratic approximation of Eq. (4) computed from Eq. (36) with the neglect of the cubic terms $\left[c_{1,2} \equiv 0\right]$ and the identification $q_{11}=q_{1}$, $q_{12}=q_{13}=q_{22}=q_{2}$. The enlarged scale of the figure shows that after Hopf bifurcation at (a) there occurs the ubiquitous period doubling cascade which begins at the $1 \rightarrow 2$ bifurcation point (b) at $\alpha=7.81$ [8.20] where for comparative purposes the value in brackets is the $1 \rightarrow 2$ bifurcation point of the exact system. The attractor in this quadratic approximation terminates finally at (c) $[\alpha=8.15]$. The effect of the quadratic approximation is to retain the period doubling scenario with a quantitative accuracy within $5 \%$ and more importantly to quench the double-scroll attractor. Within the framework of the cubic nonlinearity, the dynamics of the double scroll is essentially turned on by the cubic terms $C(x, y, z)$ in Eq. (36). The Chua circuit equations (36) exhibit an additive dynamics in that the linear terms give rise to the Hopf bifurcation, the quadratic terms dominate the period doubling scenario and the cubic terms are the cause of the double scroll. The $a b$ initio determination of the $1 \rightarrow 2$ bifurcation according to Eq. (22) involves the compounding of two approximations: neglect of the cubic terms and the approximation of the period one orbit as a simple harmonic function. The parameters of this function $D_{0,1}$ from Eq. (6b) are determined by Eq. (37). Over a range of decreasing $\beta=14,10,6$ the results are respectively 7.10 [8.20], 5.90 [6.49], 4.10 [4.50] where each number in brackets is the $1 \rightarrow 2$ bifurcation point determined by the computer solution of the exact equations. Quantitative agreement is within approximately the same error as the previous examples. The present error would be reduced by including the cubic terms in the averaging procedure. As $\beta$ decreases further the double scroll tends to be squeezed out, disappearing entirely along with the period doubling cascade at $\beta \approx 1.92$. In parallel the period two orbit disappears in the quadratic approximation as $\beta$ gets small since the second condition in Eq. (22) is no longer satisfied. These results indicate the present asymptotic method that finds the same applicability here within the same limitations above to period doubling dynamics.

\section{Discussion}

The present study has as its intent the demonstration of how a primary dynamical event, the bifurcation of a period one orbit, can be traced analytically based upon asymptotic averaging techniques. We return to the question of how general is the applicability of the present formalism to three-dimensional dynamics.

The first step of the procedure is the transformation to the equations of motion normal form (3a) and $(3 \mathrm{~b})$. For the four applications considered this could be done exactly although in the case of the Lorenz and replicator equations the form is complicated. In connection with the latter equations it is possible to effect an exact transformation from any three-dimensional Volterra system to a single third-order differential equation of the form (3a) and $(3 \mathrm{~b})$ as will be discussed elsewhere. Oppositely, the transformed equations for the Rössler equations is of simple quadratic form and those for the Chua circuit can be expressed in terms of a preassigned nonlinearity function and its derivatives. Quite generally the choice of coordinate $x$ can be any $X_{k}$, $k=1,2,3$ of the original system coordinates of Eq. (1) or any function of these coordinates [for example $x=\ln (Z)$ for the replicator equations]. The choice implies the selection of a particular Poincaré section for study which can demonstrate features of the dynamics. For example, the transformed Lorenz equations become improper at a point which divides the dynamics into chaotic and inverse period doubling regions. The Chua circuit equations with a cubic nonlinearity demonstrate a natural dynamical progression from Hopf bifurcation to period doubling cascade to double-scroll attractor. Any threedimensional system which can exactly analytically be transformed to the form (3a) and (3b) could in principle serve as a candidate for the present analysis. There are two provisos, however. First, the transformation must be proper and not ill-defined for coordinate regions vicinal to the period doubling bifurcation. Secondly, any conclusions are only for 
a Poincaré section defined by the choice of coordinate $x$.

The second step of the procedure is either truncation or emasculation of the attractor and its reconstruction in terms of the generic quadratic form in (4). This construction, being the next step after linearization, develops approximate solutions from the reference of harmonic functions. Even if the nonlinearities of the original equations are nonpolynomial so that an exact transformation to a thirdorder differential equation [Eqs. (3a) and (3b)] is not possible one might expect that expanding around the fixed point through quadratic terms would lead to the form in Eq. (4) valid in control parameter regions vicinal to a $1 \rightarrow 2$ bifurcation. In any event this quadratic approximation is compounded by the further approximation of the period one orbit as a simple harmonic function. As a consequence there must be quantitative limitations in the achievable accuracy to prediction of $1 \rightarrow 2$ control parameter points of bifurcation. The examples have indicated where these limits to quantitative prediction reside and at least in these cases the errors are easily traceable to these approximations. The validity of a simply harmonic period one function according to Eq. (6), perhaps refined to include one or more higher harmonics, and subsequent averaging according to Sec. 3 further restricts the class of systems which feature soft oscillation to the exclusion of hard relaxation oscillations. The van der Pol equations which provide the two-dimensional prototype of the latter [Jackson, 1991], are characterized by a cubic nonlinearity. One might expect that for relaxation oscillations cubic terms would dynamically dominate quadratic terms in threedimensional systems. Within the present framework this implies relaxation oscillation dynamics and bifurcations arising therefrom [Petrov et al., 1992] would require Eq. (4) be extended to include terms cubic in $[x, y, z]$.

The requirements, then, for applicability of the present formalism to bifurcation dynamics are minimally analyticity of coordinate transformation in dynamical regions vicinal to the bifurcation events and to good approximation harmonic period one structure. Beyond this, the extension of Eq. (4) to include cubic terms might be expected to encapsulate not only as discussed here the scroll attractor of the Chua circuit but also relaxation oscillations whose path to multiple periodicity and bifurcation invoke other scenarios.

\section{References}

Arneodo, A., Coullet, P. \& Tressor, C. [1980] "Occurrence of strange attractors in three-dimensional Volterra equations," Phys. Lett. A79, 259-263.

Arneodo, A., Coullet, P. H., Spielgel, E. A. \& Tresser, C. [1985] "Asymptotic chaos," Physica D14, 327-347.

Chua, L. O. [1992] "The genesis of Chua's circuit," Archiv fur Electronik und Ubertragungstechnik 46, 250-257.

Holmes, C. \& Holmes, P. [1981] "Second order averaging and bifurcations to subharmonics in Duffing's equation," J. Sound Vib. 78, 161-174.

Hofbauer, J. [1981] "On the occurrence of limit cycles in the Volterra-Lotka equation," Anal. Th. Methods Appl. 5, 1003-1007.

Hofbauer, J. \& Sigmund, K. [1988] The Theory of Evolution and Dynamical Systems (Cambridge University Press, NY).

Iooss, G. \& Joseph, D. [1990] Elementary Stability and Bifurcation Theory (Springer-Verlag, NY), Chap. XI.

Jackson, E. A. [1991] Perspectives in Nonlinear Dynamics (Cambridge University Press, NY).

Khibnik, A. I., Roose, D. \& Chua, L. O. [1993] "The periodic orbits and homoclinic bifurcations in Chua's circuit with a smooth nonlinearity," in Chua's Circuit: A Paradigm for Chaos, ed. Madan, R. N. (World Scientific, Singapore), pp. 145-178.

Krylov, N. M. \& Bogoliubov, N. N. [1947] Introduction to Nonlinear Mechanics (Princeton University Press, Princeton, NJ).

Kubiček, M. \& Marek, M. [1983] Computational Methods in Bifurcation Theory and Dissipative Structures (Springer-Verlag, NY).

Lorenz, E. N. [1963] "Deterministic nonperiodic flow," $J$. Atmos. Sci. 20, 130-141.

Madan, R. N. \& Wu, C. W. [1993] "Introduction to experimental chaos using Chua's circuit," in Chua's Circuit: A Paradigm for Chaos, ed. Madan, R. N. (World Scientific, Singapore), pp. 59-89.

Metropolis, N., Stein, M. L. \& Stein, P. R. [1973] "On finite limit sets for transformations on the unit interval," J. Combinat. Th. A15, 25-44.

Michielin, O. \& Phillipson, P. E. [1994] "Construction of Poincaré maps for multiply periodic and chaotic flows," Phys. Lett. A188, 309-316.

Michielin, O. \& Phillipson, P. E. [1997] "Map dynamics study of the Lorenz equations," Int. J. Bifurcation and Chaos 7, 373-382.

Peitgen, H.-O., Jürgens, H. \& Saupe, D. [1992] Chaos and Fractals, New Frontiers of Science (Springer-Verlag, NY).

Petrov, V., Scott, S. K. \& Showalter, K. [1992] "Mixedmode oscillations in chemical systems," J. Chem. Phys. 97, 6191-6198.

Phillipson, P. E. \& Schuster, P. [1994] "Map dynamics of 
autocatalytic networks and the replicator equations," J. Math. Biol. 32, 545-562.

Phillipson, P. E. \& Schuster, P. [1998] "Analytics of bifurcation," Int. J. Bifurcation and Chaos 8, 471-482.

Rössler, O. E. [1976] "An equation for continuous chaos," Phys. Lett. A57, 397-398.

Schnabl, W., Stadler, P. F., Forst, C. \& Schuster, P. [1991] "Full characterization of a strange attractor," Physica D48, 65-90.

Schuster, P., Sigmund, K. \& Wolff, R. [1980] "Mass action kinetics of selfreplication in flow reactors," $J$. Math. Anal. Appl. 78, 88-112.

Sparrow, C. [1982] The Lorenz Equations: Bifurcations, Chaos and Strange Attractors (Springer-Verlag, NY).

\section{Appendix Derivation of Eq. (27)}

For a selected coordinate here $Z$ the strategy is to express the coordinates $X, Y$ as functions of $x=Z$, $y=d Z / d t$. From Eq. (26a),

$$
X^{2} Y^{2}=\left[\frac{d Z}{d t}+b \varepsilon Z\right]^{2} \equiv A
$$

Then differentiation of the third equation of Eq. (26a) combined with the other two equations results in

$$
\begin{aligned}
\frac{d^{2} Z}{d t^{2}} & +\varepsilon(b+\sigma+1) \frac{d Z}{d t}+b \varepsilon^{2}(\sigma+1) Z \\
& =(\sigma-Z) X^{2}+Y^{2} \equiv B
\end{aligned}
$$

Substitution of $Y^{2}$ from Eq. (A.1) in Eq. (A.2) gives a quadratic equation in $X^{2}$, so that

$$
\begin{gathered}
X^{2}=\frac{B-\sqrt{B^{2}+4(x-\sigma) A}}{2(\sigma-x)}, \\
Y^{2}=B-(\sigma-x) X^{2}
\end{gathered}
$$

Differentiating, finally, Eq. (A.2), with the use of Eqs. (26a),

$$
\begin{aligned}
\frac{d^{2} Z}{d t^{2}}= & -\varepsilon(b+\sigma+1) \frac{d^{Z}}{d t^{2}}+4(\sigma-Z)\left[\frac{d Z}{d t}+b \varepsilon Z\right] \\
& -\left[2 \varepsilon \sigma(\sigma-Z)+\frac{d Z}{d t}\right] X^{2}-2 \varepsilon Y^{2}
\end{aligned}
$$

and substituting Eq. (A.3) into Eq. (A.4) results in Eq. (27) with the identification $[Z, d Z / d t$, $\left.d^{2} Z / d t^{2}\right] \equiv[x, y, z]$. 